\title{
Effect of Masticatory Movement Using Gum on Walking and Cycling: A Randomized Crossover Design
}

\author{
Byung-Gul Lim MSc ${ }^{1, \dagger}$, Hyunji Kim MSc ${ }^{1, \dagger}$, Jun-Hyun Bae MSc ${ }^{1}$, Ji-Won Seo MSc ${ }^{1}$, Seong-Eun Kwak MSc ${ }^{1}$, \\ Chae-Young Shin BS ${ }^{1}$, Jooeun Ahn PhD ${ }^{1,2}$, Wook Song PhD ${ }^{1,3}$ \\ ${ }^{1}$ Department of Physical Education, Seoul National University, Seoul; ${ }^{2}$ Institute of Sport Science, Seoul National University, Seoul; ${ }^{3}$ Institue on Aging, Seoul National \\ University, Seoul, Korea
}

PURPOSE: Gum chewing stimulates the sympathetic nervous system and increases energy consumption. However, the effect of mastication on physical activity remains unclear. This study aimed to investigate the effect of gum masticatory movement on physiological markers related to walking and muscle activation during cycling in different patient groups.

METHODS: Using a randomized crossover design, 25 participants participated in walking trials with a 1-week washout; the trials included chewing gum (GUM), taking a candy with the same ingredients as the gum (CAN), and no ingestion (CON). Energy expenditure (EE), metabolic equivalent (MET), oxygen consumption $\left(\mathrm{VO}_{2}\right)$, and heart rate were measured using a portable metabolic device. The walking distance was also calculated. In the cycling experiment, the other 19 participants randomly completed 7 minutes of the three trials (GUM, CAN, CON) with a 15-minute break. The mean cycling period (MCP), cycle number (CN), coefficient of variation of the cycling period $(\mathrm{CV})$, and integrated electromyography (iEMG) results were measured using the Delsys Trigno ${ }^{\mathrm{TM}}$ Wireless EMG System.

RESULTS: The walking distance was significantly higher in the GUM group than in the CAN and CON groups by an average of $78 \mathrm{~m}$ $(7.4 \%, p<.05)$. Comparing the GUM and CON groups, EE, METs, and $\mathrm{VO}_{2}$ demonstrated a partially significant increase after 15 minutes. In the cycling experiment, there were no significant differences in the effects of the trials on cycling performance (MCP, CN, CV). However, significant differences were observed in the GUM group for the iEMG results.

CONCLUSIONS: Our study results suggest that gum chewing improves physical performance, such as walking distance, and improves energy metabolism, such as EE and METs. Additionally, it can influence the improvement in the lower limb muscle activity during cycling.

Key words: Mastication, Energy expenditure, Exercise, Muscle activation, Cycle

\section{INTRODUCTION}

Obesity is an important public health problem and is associated with many serious health conditions, such as cardiovascular disease and metabolic syndrome [1,2]. For preventing the progression and onset of these problems, effective preventive methods are needed. Previous studies have reported the positive effects of masticatory movement using gum on physiological aspects. The tendency to suppress ghrelin with gum chewing indicates that mastication movement helps control food intake $[3,4]$. Additionally, gum-chewing stimulates the sympathetic nervous system and energy expenditure and increases heart rate [5-7].

The benefits of physiological factors through gum chewing could lead to increased exercise efficiency by enhancing energy expenditure $[8,9]$. Combining gum chewing and exercise increases energy expenditure more than exercise or gum chewing alone. These results suggest that exercise with masticatory movement using gum could be an effective treat-

Corresponding author: Wook Song Tel +82-2-880-7791 Fax+82-2-886-7804 E-mail songw3dasnu.ac.kr

${ }^{\dagger}$ The authors contributed to this work equally.

*This work was supported by Lotte Confectionery Co., Ltd. (Grant number: 0564-20190009).

Keywords Mastication, Energy expenditure, Exercise, Muscle activation, Cycle

Received 15 May 2021 Revised 22 Jun 2021 Accepted 7 Jul 2021

(a)This is an Open Access article distributed under the terms of the Creative Commons Attribution Non-Commercial License (https://creativecommons.org/licenses/by-nc/4.0/) which permits unrestricted non-commercial use, distribution, and reproduction in any medium, provided the original work is properly cited. 
ment and supplementary method for managing obesity.

However, the interaction between gum chewing and physical activity remains unclear. Existing studies that reported the positive effects of gum chewing during exercise to have some experimental limitations. Accordingly, our study was designed to determine the accurate effects of masticatory movement using gum on physiological markers related to walking exercise and muscle activation during the cycle.

Other studies have also indicated that voluntary clenching of the teeth can lead to increased excitability of the alpha motor neuron pool in muscles of both the upper and lower limbs [10-12]. In this study, we aimed to assess the effect of gum chewing on lower limb muscle activation during cycling and physiological factors during walking.

\section{METHODS}

This study completed two different types of tests with different groups (walking and cycling). Therefore, we explained each test separately.

\section{Participants (Demographic data, table) 1) Walking test}

Twenty-five healthy untrained participants (18 females and seven males) were included in the study, and the characteristics of the participants were as follows: age, $42.00 \pm 6.33$ years; height, $166.09 \pm 8.31 \mathrm{~cm}$, weight, $65.22 \pm 16.85 \mathrm{~kg}($ mean $\pm \mathrm{SD})$. Through pre-screening, those who exceeded the American College of Sports Medicine recommended exercise regime thrice weekly or had jaw joint disorders, insomnia, or smoked were excluded. All participants were provided with oral and written information and provided written consent for participation.

\section{2) Cycling test}

Nineteen healthy untrained participants (13 females and six males) participated in this study. The physical characteristics of the participants were as follows: age, $43.00 \pm 5.70$ years; height, $166.76 \pm 7.53 \mathrm{~cm}$, weight, $66.47 \pm 10.82 \mathrm{~kg}$ (mean \pm standard deviation, SD). The same exclusion criteria for the walking test were applied to the participants of the cycling test. They were asked to refrain from performing severe exercise before the testing day. All procedures were approved by the Institutional Review Board of Seoul National University, Seoul, Korea (IRB No. 2003/ 002-007).

\section{Study Design \\ 1) Walking test}

This study used a randomized crossover design. All participants randomly followed the same protocol that 15 minutes walking trials with three different conditions (GUM, CON, and CAN) [1,13]. Participants were randomly assigned to take a walking test with candy, control, or gum. They were asked to visit to indoor track three times for walking tests with a 1-week washout between every visit.

\section{2) Cycling test}

The participants of the cycling study also randomly completed 7 minute of three trials (GUM, CON, CAN) with a 15-minute resting period. However, participants who performed cycling, completed it in a single day for maintaining the same location as the electromyography (EMG) sensors.

\section{Procedures \\ 1) Walking test}

Participants wore a portable wireless gas analyzer (COSMED K5, Italy) on the back, a heart rate monitor (Garmin, USA) on the chest, and a mask connected to the gas analyzer on the face while walking. Energy expenditure (EE), metabolic equivalent (MET), oxygen consumption $\left(\mathrm{VO}_{2}\right)$, and heart rate $(\mathrm{HR})$ were measured using a portable device. Walking distance was also calculated manually. Before the test, the mask was checked properly after the participant took some time to adjust the chewing gum with a mask. In the control trials, participants did not ingest anything while walking at their normal pace on a $243 \mathrm{~m}$ indoor track for 15 minutes. In gum trials, they began chewing three pellets of gum $(5 \mathrm{~g}, 0.2 \mathrm{kcal}) 30$ seconds before the walking test was initiated and kept chewing gum while walking. In candy trials, they took a piece of candy ( $1 \mathrm{~g}, 0.2 \mathrm{kcal})$, with the same ingredients as gum, 30 seconds before the walking test was initiated and were asked not to chew it, but melt it. Gum and candy were prepared for use in the experiments by Lotte Confectionery Co., Ltd. Gum consists of xylitol; gum base (acesulfame potassium, sucralose); D-sorbitol; synthetic flavor (apple mint, apple, and menthol); Arabia gum; rienamel I (calcium phosphate, CPP, and funoran); mixed formulation (shellac, processed fat); emulsifier; carnauba wax; and methyl hesperidin. Candy was composed of the same ingredients, except for the gum base. Participants performed a total of three walking tests with a 1-week washout period simultaneously under controlled environmental conditions, including temperature $\left(18 \pm 1.1^{\circ} \mathrm{C}\right)$ and 
humidity $(42 \pm 3.3 \%)$. Participants maintained a normal diet and activities of daily living, abstained from smoking, alcohol, extraneous exercise, and restricted caffeine for a minimum of 24 hours before the trial. Walking tests were performed individually, separately, on an indoor track of $243 \mathrm{~m}$

\section{2) Cycling test}

The following three conditions were tested, as in the previous walking experiment: (1) cycling only (CON), (2) cycling while chewing three gums (GUM), and (3) cycling while taking the melting candy (CAN). Each participant was instructed to cycle for 7 minutes continuously at their preferred revolutions per minute on a cycle ergometer. A rest of 15 minutes was allowed between trials. Cycling was performed using a cycle ergometer (Lode, Groningen, Netherlands). The cycle ergometer recorded the total cycling distance for 7 minutes. At the beginning of each experiment, the height of the saddle was adjusted to match the height of the participant's anterior superior iliac spine. The mechanical power was set at $60 \%$ of the weight of the participant for maintaining low-mediumintensity aerobic exercise. The surface EMG electrodes (Delsys, Inc., Boston, MA, USA) were positioned on the tibialis anterior (TA; dorsiflexor of the ankle), Gastrocnemius medialis (GM; plantar flexor of the ankle), Vastus lateralis (VL; extensor of the knee), and Biceps femoris (BF; flexor of the knee) of both legs. The EMG signal was recorded using a data acquisition system (Delsys, Inc.) at a sampling rate of 2,000 Hz. The raw EMG signals were digitized at $1,000 \mathrm{~Hz}$ and amplified. The EMG signals were bandpass filtered (fourth-order Butterworth) at 10-200 Hz. Each participant's cycling performance was acquired using a Delsys triaxial accelerometer. Cycling acceleration data were collected at $72 \mathrm{~Hz}$ using the Delsys Trigno system (Delsys, Inc.). All signal processing was performed using MATLAB (MathWorks, Inc., Natick, MA, USA). All data collected during the cycling tasks were divided into 14 sets, each of which was 30 seconds long. The first set was regarded as the adaptation period and excluded; the remaining 13 sets were used for analysis. The following cycling variables were calculated for each 30 seconds set for each participant.

(1) Mean cycling period (MCP): For computing the cycling period, we extracted the maximum peaks of the acceleration $\mathrm{A}_{z}$ (vertical direction) signals from the sensor attached to the left VL. The cycling period was calculated as the interval between two neighboring maximum peaks of $A_{z}$. The mean value of the cycling period in each 30 seconds set was obtained.
(2) Cycle number $(\mathrm{CN})$ : The number of cycles was the number of maximum peaks of the acceleration $\mathrm{A}_{\mathrm{z}}$ obtained from deriving the mean cycle time.

(3) Coefficient of variation $(\mathrm{CV})$ of the cycling period: The $\mathrm{CV}$ of the cycling period is the ratio of the standard deviation (SD) of the period to the mean period. $(\mathrm{CV}[\%]=100 \times \mathrm{SD} /$ mean $)$

(4) Integrated electromyography (iEMG) data: The EMG data were processed using a root-mean-square with a $12 \mathrm{~ms}$ moving window. Then, iEMG was calculated during each period and divided by the average value of the control trial. The average ratio across each period was then calculated.

\section{Statistical Analysis}

Data are presented as the mean \pm standard error of the mean. The sample size was calculated using a power and sample size program that based on a power 0.80 , significance $p=.05$, and the effect size of 0.75 a minimum sample size of 21 was estimated. One-way analysis of variance (ANOVA) was used to compare walking distance. For comparing METs per minute, EE per minute, and HR per minute between trials, two-way ANOVA was used. In addition, the METs and EE of 12 participants whose walking distance in a gum trial increased were non-normally distributed; therefore, they were analyzed using the Friedman test, a nonparametric statistical test. Statistical significance was set at $p<.05$. The data were analyzed using SPSS 23.0 (SPSS Inc., USA).

In the cycling experiment, two-way repeated (trial $\times$ set [time]) ANOVA, with conditions and cycling performance data as main effects followed by a Tukey's test was applied to the acceleration data. For comparing normalized iEMG data between trials, one-way ANOVA was used Statistical significance was set at $p<.05$. The data were analyzed using SPSS (version 23.0; SPSS Inc., USA).

\section{RESULTS}

\section{Masticatory movement by gum upregulate walking performance}

For determining whether gum chewing affects walking performance, we measured each participant's walking distance for 15 minutes, with or without gum. There was a significant change in the walking distance (Fig. 1A). The total walking distance was higher in the GUM group than in the CON and CAN groups ( $p<.05)$. It was improved by $7.34 \%(78 \mathrm{~m})$ and $6.74 \%(72 \mathrm{~m})$ in the GUM group compared with those in the CON 

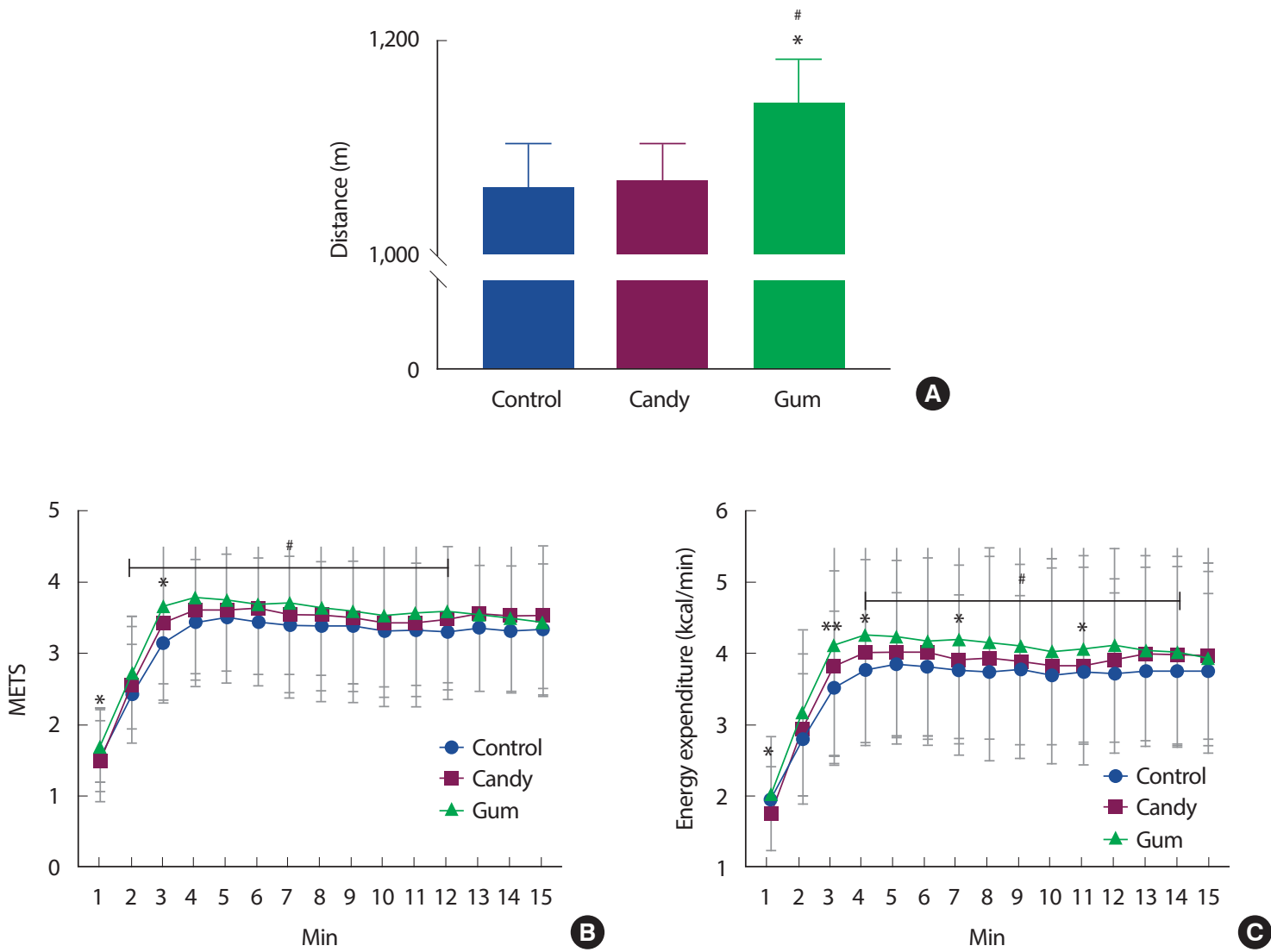

Fig. 1. Masticatory movement by gum effects on total walking distance and energy metabolism function index. The figure shows each group's mean of walking distance ( $\mathrm{N}=25$, each group) and energy metabolism function index changes during 15 minutes of experiment. ${ }^{*} p<.05$, ${ }^{*} p<.01, \mathrm{CAN}$ vs. GUM., $" p<.05$, CON vs. GUM. The statistical analysis was performed using Repeated-measured One-way ANOVA and Tukey's multiple comparisons test. Data are means \pm SEMs.

and CAN groups, respectively. This indicates that chewing gum improves walking performance.

\section{Effects of masticatory movement using gum for 15 minutes on energy metabolism function index}

For determining the effects of gum chewing on energy metabolism by exercise duration, we analyzed METs and energy expenditure every minute. There was a significant change in the METs and EE results (Fig. 1A, C). From 2 minutes to 12 minutes, the GUM group showed significantly higher METs than the CON group $(p<.05)$. In addition, from 4 minutes to 14 minutes, the GUM group showed significantly higher EE than the CON group $(p<.05)$. METs in GUM for 1 minute and 3 minutes were markedly increased by mastication compared with CAN. EE in GUM for $1,3,4,7$, and 11 minutes was markedly increased by mastication compared with CAN.

\section{METs and EE among participants who significantly increased total walking distance}

As associations existed between total walking distance and physiological factors, we next determined whether there was a direct effect of mastication movement using gum among participants who resulted in increased total walking distance. METs were significantly increased in the GUM group compared to those in the CON and CAN groups $(p<.05)$. In addition, EE was significantly higher in the GUM group than in the CON group $(p<.05)$ (Fig. 2A, B).

\section{Gum chewing effects on cycling performance (speed, mean cycling period, mean cycle number, $\mathrm{CV}$ )}

For determining whether gum chewing affects cycling performance, we measured each participant's cycling distance and average speed for 7 minutes with three trials (conditions). There was no significant change in the speed results (Fig. 3A); the difference, if any, could not be identified by the coarse resolution of the bike erg, which was $100 \mathrm{~m}$. Among 

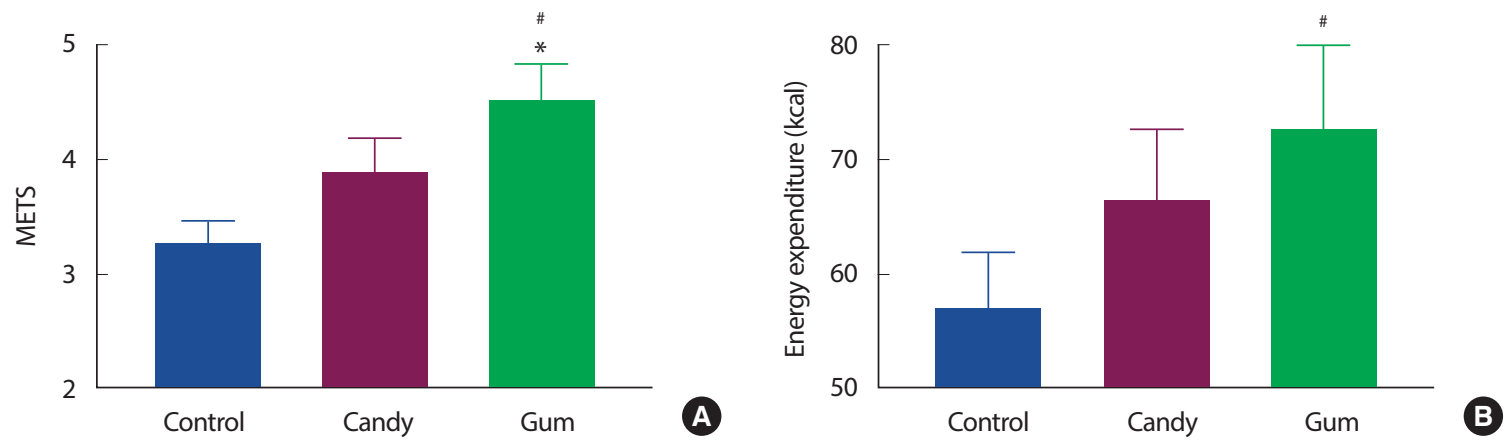

Fig. 2. Gum chewing upregulates METs and energy expenditure. Figures show METS (A) and energy expenditure (B) difference between each group who have shown upregulation of walking distance by gum chewing ( $\mathrm{N}=25$, each group). ${ }^{*} p<.05$, CAN vs. GUM. ${ }^{*} p<.05$, CON vs. GUM. The statistical analysis was performed using Repeated-measured One-way ANOVA and Tukey's multiple comparisons test. Data are means \pm SEMs.
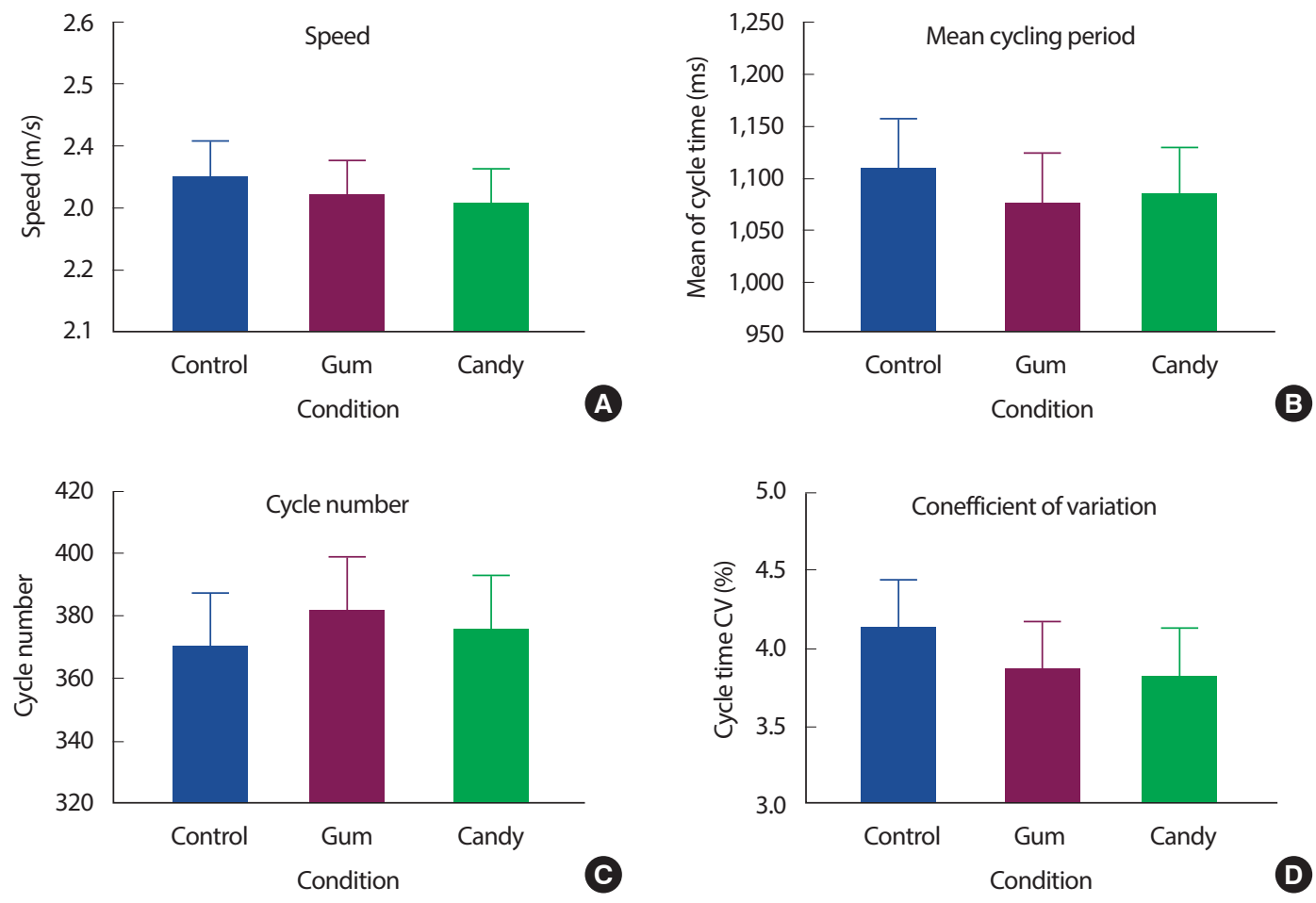

Fig. 3. Gum chewing effects on cycling performance. Gum chewing effects on Speed in (A), effects on mean cycling period in (B), effects on cycle number in (C), effects on CV in (D). Mean values with SEM bars are shown. GUM is the lowest mean cycling period, highest cycle number, lowest CV with non-significant differences $(p<.05)$.

the three conditions, the GUM group showed the least cycling period; however, the difference was not statistically significant (Fig. 3B). Accordingly, the GUM group also showed the highest cycling number; however, without statistical significance (Fig. 3C). The CV of the cycling period was an indicator of the pedaling rhythm constant. Referring to a previous study, constant rhythm provided positive feedback on maintaining stability during gait, reducing the incidence of muscular fatigue. Both GUM and CAN conditions showed a lower CV, however, without statistical significance (Fig. 3D).
In summary, there were no statistically significant results.

\section{Gum chewing effects on the pattern of lower limb muscle activation during cycling (normalized iEMG)}

In the GUM group, normalized iEMG values of the left leg showed statistically higher values in the four lower limb muscles than in the CAN and CON groups $(p<.001)$. There was no significant difference in the normalized iEMG values between the CAN and CON group (Fig. $4 \mathrm{~A})$. On the right leg, normalized iEMG values of the GUM group dem- 

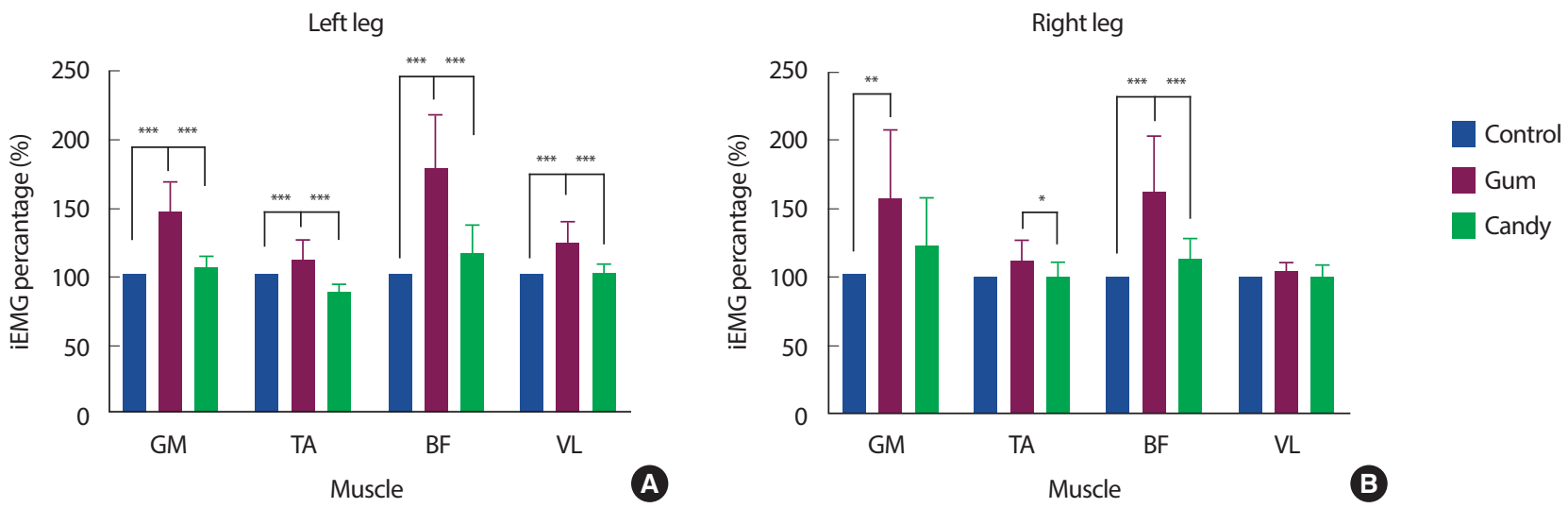

Fig. 4. Gum chewing effects on pattern of lower limb muscle activation during cycling (normalized iEMG). The normalized iEMG data on the left leg in (A), and the data on the right leg in (B). Mean values with standard deviation bars are shown. The statistical analysis was performed using two-way ANOVA and Tukey's multiple comparisons test. Data are means \pm SEMs. GM, Gastrocnemius; Ta, Tibialis anterior; BF, Biceps femoris; VL, Vastus lateralis. ${ }^{*} p<.05$, ${ }^{* *} p<.01$, $* * * 0<.001$.

onstrated statistically higher values in GM $(p<.01)$ and BF $(p<.001)$ muscles than those of the CON group. Normalized iEMG values of the GUM group showed statistically higher values in TA $(p<.05)$ and BF $(p<.001)$ muscles than those of the CAN group. There was no significant difference in normalized iEMG values in VL muscle and four lower limb muscles between the CAN and CON groups similar to the left leg. (Fig. 4B) These suggest that chewing gum significantly helped activate all motor neuron pool of lower limb muscles during cycling.

\section{DISCUSSION}

This study was conducted to confirm the effectiveness of chewing gum. First, we hypothesized that chewing gum would improve physical performance, such as walking and cycling distance. Furthermore, to determine the effects of mastication on physical performance, we compared the gum chewing and candy groups. Second, we hypothesized that chewing gum would improve the energy metabolism index. We found that chewing gum improved both physical performance and energy metabolism index. Third, we hypothesized that chewing gum would influence the muscle activation lower limb during rhythmic action, such as cycling. We observed that mastication movement could effect on the pattern of lower limb muscle activation through the signal of normalized iEMG.

As aforementioned, unlike previous studies, the addition of the candy group allowed us to identify the effects of mastication activity on physical performance during walking and cycling. Also, no other published articles were found concerning the effect of mastication on lower limb muscle activation.

The effects of chewing gum on physical performance and energy metabolism index were consistent with those of a previous study in Japan $[1,13]$. Also, the rhythmical mastication could be influenced on an individual's gait [11]. However, the results of the candy group were in the middle of the control group and the gum-chewing group, and it seems that only the component of the gum can improve the physical performance and energy metabolism index. The candy and gum were mainly composed of xylitol, and a previous study showed that xylitol can retain moisture; hence, this might prevent oral dehydration [14]. Additionally, xylitol could benefit microorganisms such as health-promoting bacteria, glucose levels, and blood lipids [14-16]. Therefore, these benefits of xylitol may affect physical performance and the energy metabolism index.

We also observed that the energy expenditure level was upregulated by gum chewing. A previous study showed that gum chewing spends $4-5 \mathrm{kcal} / \mathrm{min}$ of energy [9], and mastication affects the activation of the sympathetic nervous system [15]. The sympathetic nervous system is related to energy expenditure [5,6]; therefore, mastication can affect energy expenditure through the activation of the sympathetic nervous system. These results are consistent with the METs and other energy metabolism indices. Additionally, a previous study showed that the positive effects of mastication on EE were mostly associated with aerobic exercise.

We also analyzed the energy metabolism function index of patients with improved walking distance using chewing gum. Consequently, we found that those who had improved walking distance showed significant upregulation of the energy metabolism function index by gum chewing This result indicates that gum mastication has a positive effect on our 
body, which is consistent with previous studies [1-4,8,9,13].

In the cycling study, we analyzed the impact of gum chewing on cycling performance. We found that there was no significant improve in cycling performance (speed, MCP, CN, and CV) when chewing gum. In this study, we asked the participants to cycle a bike ergometer, chewing gum at a preferred pace. A previous study showed that the rate at which a person chewed and the stepping rate has a strong driving influence, called the coupling of motor oscillators [11]. Thus, it seems that the results of the cycling performance varied slightly with some gum chewing pace. Therefore, further studies should be conducted at a consistent gum-chewing pace or under conditions of tracking the gum-chewing pace. However, irrespective of the gum-chewing pace, mastication itself showed a positive effect on the muscle activity of the lower limbs during cycling. We observed that gum chewing conditions showed significantly higher muscle activity of the lower limbs (especially in the BF and GM) than in the candy or control conditions. This is consistent with previous studies showing that mastication can lead to an increase in the neural drive and excitability of the alpha motor neuron pool for muscles of both the upper and lower limbs $[5,11,12]$. However, previous masticationrelated studies have been conducted simply in standing or standing balance or walking situations and showed only one lower limb muscle such as the tibialis anterior (TA) $[5,11,17]$. In this study, even in a more dynamic task, the same neural mechanisms for the increased excitation of the various lower extremities used for the task were demonstrated during gum chewing.

This study showed gum-chewing effects on various health-related function indices, but participants did not take same food before each walking test and these were limited to walking and cycling, which is a type of low-intensity exercise. Therefore, further studies should be performed to analyze gum-chewing effects at various intensities of exercise. Gum-chewing effects during various intensities of exercise might clarify the effects of mastication on exercise performance and energy metabolism.

In conclusion, our study results suggest that gum chewing improves physical performance such as walking distance, and also improves energy metabolism, such as energy expenditure and METs. Additionally, it can influence the improvement in lower limb muscle activity during cycling. This study was limited to a small number of participants and a few measuring methods. However, based on these results, it is recommended that gum chewing while walking or cycling increases energy expenditure and affects muscle activity and rhythm of cycling performance.

\section{CONFLICT OF INTEREST}

The authors declare that the study was conducted in the absence of any commercial or financial relationships that could be construed as a potential conflict of interest.

\section{AUTHOR CONTRIBUTIONS}

Conceptualization: BG Lim; Data curation: SE Kwak, CY Shin; Formal analysis: SE Kwak, BG Lim, H Kim; Funding acquisition: W Song; Methodology: JH Bae, JW Seo; Project administration: BG Lim, W Song; Visualization: CY Shin, H Kim; Writing-original draft: BG Lim, H Kim; Writing-review \& editing: W Song, J Ahn.

\section{ORCID}

$\begin{array}{ll}\text { Byung-Gul Lim } & \text { https://orcid.org/0000-0002-4819-7370 } \\ \text { Hyunji Kim } & \text { https://orcid.org/0000-0002-2896-9329 } \\ \text { Jun-Hyun Bae } & \text { https://orcid.org/0000-0003-1918-9213 } \\ \text { Ji-Won Seo } & \text { https://orcid.org/0000-0003-0279-7562 } \\ \text { Seong-Eun Kwak } & \text { https://orcid.org/0000-0002-3666-2618 } \\ \text { Chae-Young Shin } & \text { https://orcid.org/0000-0002-9030-1362 } \\ \text { Jooeun Ahn } & \text { https://orcid.org/0000-0002-7964-5148 } \\ \text { Wook Song } & \text { https://orcid.org/0000-0002-8825-6259 }\end{array}$

\section{REFERENCES}

1. Kanno S, Shimo K, Ando T, Hamada Y, Miyashita M, et al. Gum chewing while walking increases fat oxidation and energy expenditure. J Phys Ther Sci. 2019;31:435-9.

2. Park E, Edirisinghe I, Inui T, Kergoat S, Kelley M, et al. Short-term effects of chewing gum on satiety and afternoon snack intake in healthy weight and obese women. Physiol Behav. 2016;159:64-71.

3. Foster-Schubert KE, McTiernan A, Frayo RS, Schwartz RS, Rajan KB, et al. Human plasma ghrelin levels increase during a one-year exercise program. J Clin Endocrinol Metab. 2005;90:820-5.

4. Li J, Zhang N, Hu L, Li Z, Li R, et al. Improvement in chewing activity reduces energy intake in one meal and modulates plasma gut hormone concentrations in obese and lean young chinese men. Am J Clin Nutr. 2011;94:709-16.

5. Boroojerdi B, Battaglia F, Muellbacher W, Cohen LG. Voluntary teeth 
clenching facilitates human motor system excitability. Clin Neurophysiol. 2000;111:988-93.

6. Passatore M, Roatta S. Modulation operated by the sympathetic nervous system on jaw reflexes and masticatory movement. Arch Oral Biol. 2007;52:343-6.

7. Takeuchi N, Ekuni D, Tomofuji T, Morita M. Relationship between masticatory performance and heart rate variability: a pilot study. Acta Odontol Scand. 2013;71:807-12.

8. Kresge DL, Melanson K. Chewing gum increases energy expenditure before and after controlled breakfasts. Appl Physiol, Nutr Metab. 2015; 40:401-6.

9. Levine J, Baukol P, Pavlidis I. The energy expended in chewing gum. N Eng J Med. 1999;341:2100.

10. Miyahara T, Higaya N, Ohyma T, Nakamura Y. Modulation of human soleus $\mathrm{H}$ reflex in association with voluntary clenching of the teeth. J Neurophysiol. 1996;76:2033-41.

11. Samulski B, Prebor J, Armitano C, Morrison S. Coupling of motor oscillators-what really happens when you chew gum and walk? Neuro- sci Lett. 2019;698:90-6.

12. Sugawara K, Furubayashi T, Takahashi M, Ni Z, Ugawa Y, et al. Remote effects of voluntary teeth clenching on excitability changes of the human hand motor area. Neurosci Lett. 2005;377:25-30.

13. Hamada Y, Yanaoka T, Kashiwabara K, Kurata K, Yamamoto R, et al The effects of gum chewing while walking on physical and physiological functions. J Phys Ther Sci. 2018;30:625-9.

14. Fukazawa T, Smyrnioudis I, Konishi M, Takahashi M, Kim HK, et al. Effects of Chios mastic gum and exercise on physical characteristics, blood lipid markers, insulin resistance, and hepatic function in healthy Japanese men. Food Sci Biotechnol. 2018;27:773-80.

15. Kaur N, Gupta AK. Applications of inulin and oligofructose in health and nutrition. J Biosci. 2002;27:703-14.

16. Ur-Rehman S, Mushtaq Z, Zahoor T, Jamil A, Murtaza MA. Xylitol: a review on bioproduction, application, health benefits, and related safety issues. Crit Rev Food Sci Nutr. 2015;55:1514-28.

17. Kushiro K, Goto F. Effect of masticating chewing gum on postural stability during upright standing. Neurosci Lett. 2011;487:196-8. 